\title{
CORRIGENDUM
}

\section{Maternal overweight and obesity and risk of congenital heart defects in offspring}

J Brite, SK Laughon, J Troendle and J Mills

International Journal of Obesity (2014) 38, 886; doi:10.1038/ijo.2014.47

Correction to: International Journal of Obesity (2014) 38, 878-882; doi:10.1038/ijo.2013.244

The original version of this article contained two errors. In the Abstract, the correct odds ratio (OR) and 95\% confidence interval (Cl) for obese women for having an infant with conotruncal defects was $\mathrm{OR}=1.33(1.03-1.72)$, not $1.34(1.04-1.72)$. In the
Results section, the correct $\mathrm{Cl}$ for obese women at the site that collected OGTT results, after adjusting for age and OGT, was 1.12-5.05, not 1.02-3.76.

The corrected article appears in this issue and the html and online pdf versions have also been amended. The authors would like to apologize for these errors. 